\title{
HST spectra reveal accretion in MY Lupi ${ }^{\star}$
}

\author{
J. M. Alcalá ${ }^{1}$, C. F. Manara ${ }^{2}$, K. France ${ }^{3}$, C. P. Schneider ${ }^{4}$, N. Arulanantham ${ }^{3}$, A. Miotello ${ }^{2}$, \\ H. M. Günther ${ }^{5}$, and A. Brown ${ }^{6}$
}

\author{
${ }^{1}$ INAF - Osservatorio Astronomico di Capodimonte, Via Moiariello 16, 80131 Napoli, Italy \\ e-mail: juan.alcala@inaf.it \\ 2 European Southern Observatory, Karl-Schwarzschild-Str. 2, 85748 Garching bei Munchen, Germany \\ ${ }^{3}$ Laboratory for Atmospheric and Space Physics, University of Colorado, 392 UCB, Boulder, CO 80303, USA \\ ${ }^{4}$ Hamburger Sternwarte, Gojenbergsweg 112, 21029 Hamburg, Germany \\ ${ }_{6}^{5}$ MIT, Kavli Institute for Astrophysics and Space Research, 77 Massachusetts Avenue, Cambridge, MA 02139, USA \\ ${ }^{6}$ Center for Astrophysics and Space Astronomy, University of Colorado, Boulder, CO 80309-0389, USA
}

Received 10 April 2019 / Accepted 12 August 2019

\begin{abstract}
The mass accretion rate is a crucial parameter for the study of the evolution of accretion discs around young low-mass stellar and substellar objects (YSOs). We revisit the case of MYLup, an object where VLT/X-shooter data suggested a negligible mass accretion rate, and show it to be accreting on a level similar to other Class II YSOs in Lupus based on Hubble Space Telescope (HST) observations. In our HST-Cosmic Origins Spectrograph (HST-COS) and -Space Telescope Imaging Spectrograph (HST-STIS) spectra, we find many emission lines, as well as substantial far-ultraviolet (FUV) continuum excess emission, which can be ascribed to active accretion. The total luminosity of the C IV $\lambda 1549 \AA$ doublet is $4.1 \times 10^{-4} L_{\odot}$. Using scalings between accretion luminosity, $L_{\text {acc }}$, and C IV luminosity from the literature, we derive $L_{\text {acc }} \sim 2 \times 10^{-1} L_{\odot}$, which is more than an order of magnitude higher than the upper limit estimated from the X-shooter observations. We discuss possible reasons for the X-shooter-HST discrepancy, the most plausible being that the low contrast between the continuum excess emission and the photospheric+chromospheric emission at optical wavelengths in MY Lup hampered detection of excess emission. The luminosity of the FUV continuum and C IV lines, strong $\mathrm{H}_{2}$ fluorescence, and a "1600 A Bump" place MY Lup in the class of accreting objects with gas-rich discs. So far, MY Lup is the only peculiar case in which a significant difference between the HST and X-shooter $\dot{M}_{\text {acc }}$ estimates exists that is not ascribable to variability. The mass accretion rate inferred from the revisited $L_{\text {acc }}$ estimate is $\dot{M}_{\text {acc }} \sim 1\left({ }_{-0.5}^{+1.5}\right) \times 10^{-8} M_{\odot} \mathrm{yr}^{-1}$. This value is consistent with the typical value derived for accreting YSOs of similar mass in Lupus and points to less clearing of the inner disc than indicated by near- and mid-infrared observations. This is confirmed by Atacama Large Millimeter Array (ALMA) data, which show that the gaps and rings seen in the sub-millimetre are relatively shallow.
\end{abstract}

Key words. stars: pre-main sequence - stars: low-mass - accretion, accretion disks - stars: individual: MY Lupi protoplanetary disks

\section{Introduction}

A key issue in the study of planet formation is to explain how optically thick accretion discs surrounding the youngest lowmass $\left(M_{\star} \lesssim 2.0 M_{\odot}\right)$ stars evolve into optically thin debris discs. The mass accretion rate onto a young star, $\dot{M}_{\text {acc }}$, is a crucial parameter for the study of the evolution of accretion discs around young low-mass stellar and substellar objects (YSOs), because it sets important constraints for disc-evolution models (Hartmann et al. 1998, 2016) and disc-clearing mechanisms (Alexander et al. 2014; Ercolano \& Pascucci 2017, and references therein), and is a key quantity for the studies of pre-main sequence (PMS) stellar evolution and planet formation (Morbidelli \& Raymond 2016).

Transition discs (TDs) are protoplanetary discs that show evidence of inner holes and gaps, as observed in millimetre interferometric observations (e.g. Andrews et al. 2011, 2018;

\footnotetext{
* Based on observations made with the NASA/ESA Hubble Space Telescope, obtained from the data archive at the Space Telescope Science Institute. STScI is operated by the Association of Universities for Research in Astronomy, Inc. under NASA contract NAS 5-26555. Also based on observations collected at the European Southern Observatory at Paranal, under programs 095.C-0134(A), and archive data of programmes 093.C-0476(A) and 093.C-0506(A).
}

van der Marel et al. 2018) and in the dip of the mid-infrared spectral energy distribution (MIR; SED; e.g. Merín et al. 2008). Low-mass PMS stars with transitional discs accreting at very low rates are likely in the final stages of inner disc evolution, and have probably already formed proto-planets (Owen \& Clarke 2012). Therefore, identifying and investigating such slow accretors may help us to understand planet formation. However, measurements of low $\dot{M}_{\text {acc }}$ are challenging. In practice, $\dot{M}_{\text {acc }}$ can be derived from the energy released in the accretion shock (accretion luminosity $L_{\text {acc }}$, see Gullbring et al. 1998; Hartmann 1998) given the stellar properties. Observationally, this requires measurements of excess flux in continuum and lines with respect to similar nonaccreting stars. Such measurements are best performed at UV wavelengths $(\lambda \lesssim 4000 \AA$ ), with the Balmer continuum excess emission and the Balmer jump being more easily seen in the spectra of late-type (>K5) YSOs than in the early types due to the higher contrast between photospheric emission and continuum emission (Herczeg \& Hillenbrand 2008). Weak accretion, in general, is not easily detectable in the region of the Balmer jump. Some previous studies attempted $\dot{M}_{\text {acc }}$ measurements in TDs using other tracers. In the case of CVSO 224, a 10 Myr old PMS star with a TD, Espaillat et al. (2008) derived $\dot{M}_{\text {acc }}=7 \times$ $10^{-11} M_{\odot} \mathrm{yr}^{-1}$ based on modelling of the $\mathrm{H} \alpha$ emission line 
observed at high spectral resolution. Other estimates of $\dot{M}_{\text {acc }}$ in samples of YSOs with TDs have been provided using the width, $\mathrm{WH} \alpha(10 \%)$, of the $\mathrm{H} \alpha$ line measured at the $10 \%$ of the line peak in intermediate-resolution spectra (Merín et al. 2010; Cieza et al. 2010). However, such estimates heavily depend on rather uncertain scaling relations between the line width and $\dot{M}_{\text {acc }}$ (see discussion in Alcalá et al. 2014).

We measured the UV excess continuum emission for a $90 \%$ complete sample of YSOs in the Lupus star-forming region (Alcalá et al. 2014, 2017) using the VLT/X-shooter spectrograph (Vernet et al. 2011). This dataset allowed us to derive the stellar and accretion parameters in a self-consistent and homogeneous way (see also Manara et al. 2013a, for the methods). In the sample of 81 systems, 12 are YSOs with TDs based on the dip of their MIR SEDs (Merín et al. 2008; Romero et al. 2012; Bustamante et al. 2015). Among these, we identified 5 objects (Lup 607, MY Lup, Sz65, Sz68, and SST c2dJ160830.7-382827) in which the UVB excess emission, ascribable to accretion, is barely evident. The analysis of the emission lines in the X-shooter spectra of these objects showed that their excess emission is close to the chromospheric noise level as defined by Manara et al. (2013b, 2017a, see also Ingleby et al. 2011). We therefore classified them as weak or non-accretors. Two of these (MY Lup and SST c2dJ160830.7-382827) were previously classified as transitional discs and their spectral type is earlier than K5. It was thus unclear whether the apparent low level of accretion in these objects is real or due to a very low contrast between the continuum excess emission and the photospheric+chromospheric emission, which prevented a reliable assessment of accretion.

Observations in the far-ultraviolet (FUV) are particularly well suited for studies of accretion. Previous observations in the FUV have shown strong accretion-related emission features. For instance, strong emission of CO (e.g. France et al. 2011; Schindhelm et al. 2012) and $\mathrm{H}_{2}$ ascribed to gas in the inner regions ( $r \lesssim 10 \mathrm{AU})$ of the disc of accreting YSOs (Johns-Krull et al. 2000; Herczeg et al. 2002; Calvet et al. 2004; France et al. 2011, 2012, 2017; Ingleby et al. 2011; Yang et al. 2012; Hoadley et al. 2015; Arulanantham et al. 2018, and references therein). This is of particular importance for the studies of accretion in YSOs because these molecules trace the gas in the inner disc regions, indicating that molecular material persists within a few astronomical units of the star. In this work, we use UV-Hubble Space Telescope (HST) spectra to unambiguously confirm and investigate the accretion properties of MYLup. In a forthcoming paper (Arulanantham et al., in prep.), an extended set of the same data is used to study the structure and distribution of the gas in the inner disc regions. In Sect. 2 we present an overview of MY Lup and previous spectroscopic observations used to provide intial estimates of mass accretion rate, $\dot{M}_{\text {acc. }}$. In Sect. 3 we report the HST observational data, their processing, and the main spectroscopic features in the near-ultraviolet (NUV) and FUV. In Sect. 4 we describe the analysis of the HST spectra and report the results on the accretion properties of MY Lup. In Sect. 5 we discuss the implications of the assessment of accretion in MY Lup and its disc. Finally, we present our conclusions in Sect. 6.

\section{The target}

MY Lup was first classified as a T Tauri star by Gregorio-Hetem et al. (1992) based on the presence of the $\mathrm{H} \alpha$ emission line and the $\mathrm{Li}$ I $\lambda 6708 \AA$ absorption line in an intermediate resolution spectrum. It is a K0-type star with a mass of $1.1 M_{\odot}$ (Alcalá et al. 2017; Frasca et al. 2017) in the Lupus IV cloud (Comerón 2008), surrounded by a highly inclined $\left(\sim 73^{\circ}\right)$ disc (Ansdell et al. 2016; van der Marel et al. 2018; Andrews et al. 2018; Huang et al. 2018). Romero et al. (2012) classified MY Lup as a planetforming TD disc candidate, claiming that its SED could be explained by a discontinuity in the grain size distribution rather than an inner opacity hole. Recent high-contrast high-resolution imaging in the near-infrared (NIR) with the Spectro-Polarimetric High-contrast Exoplanet REsearch (SPHERE) instrument by Avenhaus et al. (2018) confirmed a high inclination of $\sim 77^{\circ}$ of the dusty disc. The SPHERE observation also revealed a flared-truncated disc structure with multiple rings on the surface, and the Disk Substructures at High Angular Resolution Project (DSHARP; Andrews et al. 2018) also detected several annular substructures at 8,20,30, and 40 au from the central star (Huang et al. 2018), with no evidence of a large inner cavity.

The age of the relatively extincted $\left(A_{\mathrm{V}}=1.3 \mathrm{mag}\right.$, Alcalá et al. 2017) star has been estimated at about 17 Myr (cf. Alcalá et al. 2017; Frasca et al. 2017), that is, a factor of six older than typical YSOs in Lupus, suggesting that the object may be more evolved than other Lupus YSOs or that the highly inclined disc occults part of the stellar light, making the object sub-luminous on the HR diagram. A tentative stellar rotational period of 2.5 days has been reported in Batalha et al. (1998).

\subsection{Previous spectroscopy and $\dot{M}_{a c c}$ estimates}

Previous VLT spectroscopy of MY Lup includes two X-shooter spectra, one by us (reported in Alcalá et al. 2017) and the other one obtained within ESO programme 095.C-0134 (PI: Caceres). In addition, a UVES spectrum was acquired within ESO programme 093.C-0476 (PI: Canovas). The latter was performed 6 days after the Caceres X-shooter observation, while our X-shooter spectrum was acquired about 1 yr later. The ESO Phase-3 reduced data of both the Caceres X-shooter and Canovas-UVES spectra are used here for comparison purposes.

Except for $\mathrm{H} \alpha$ and the $\mathrm{Ca}$ II $\mathrm{H} \& \mathrm{~K}$ and infra-red triplet (IRT) emission lines, which appear superimposed on the corresponding photospheric absorption, no other emission lines are clearly seen in the VLT spectra of MY Lup. In both the X-shooter spectra the $\mathrm{He} \mathrm{I} \lambda 1082.9 \mathrm{~nm}$ appears as a very strong absorption with a possible but barely detectable emission, resembling a P Cyg profile. When subtracting a photospheric template, still no other emission lines are evident; only the $\mathrm{Pa} \gamma, \mathrm{Pa} \beta$, and $\mathrm{Br} \gamma$ lines are barely seen, but detected (the fluxes are given in Frasca et al. 2017). A possible explanation for why we see the lines in the red but no other lines in the blue may be that the highly inclined disc occults some of the emitting inner regions, with the lines bluer than $\mathrm{H} \alpha$ being more extincted and therefore remaining undetected.

We highlight the fact that the $\mathrm{H} \alpha$ line shows a complex profile, several hundred $\mathrm{km} \mathrm{s}^{-1}$ wide (cf. Fig. 1). The width at $10 \%$ of the line peak measured in the UVES spectrum is $\sim 500 \mathrm{~km} \mathrm{~s}^{-1}$. This value is consistent with the one estimated by Romero et al. (2012) using other spectra. We also note that the X-shooter spectrum we used in Alcalá et al. (2017) has the weakest $\mathrm{H} \alpha$ emission.

Variability of the $\mathrm{H} \alpha$ line profile is observed on a timescale of $1 \mathrm{yr}$, but some variations are detected within a few days (see Fig. 1). The type of profile is consistent with the model predictions by Kurosawa et al. (2006, see their Fig. 13) for a high-inclination angle of accreting sources. In those models, the absorption feature becomes stronger as the inclination increases, mainly because of the geometrical configuration. A high inclination is in agreement with the disc inclination estimated from the Atacama Millimeter Array (ALMA) millimetre 


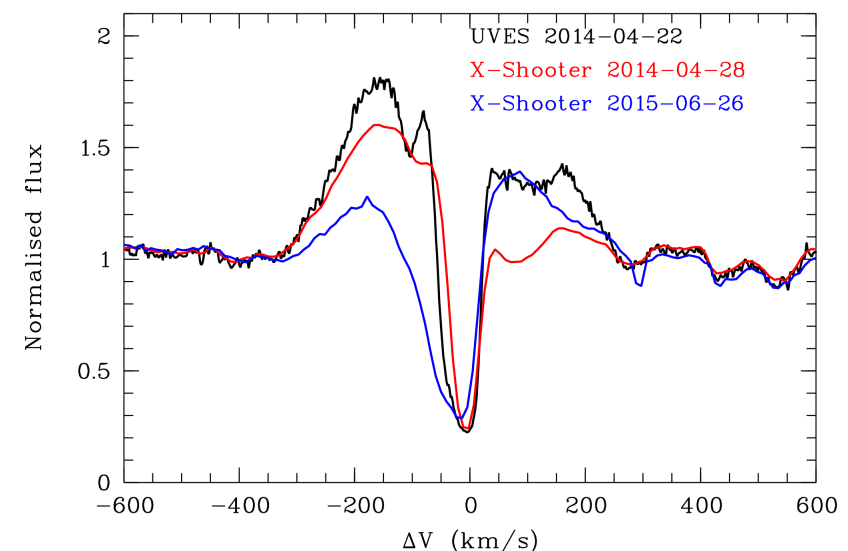

Fig. 1. $\mathrm{H} \alpha$ line profile of MY Lup observed in three epochs as labelled. The spectra are normalised to unity and plotted in velocity scale.

(Ansdell et al. 2016; van der Marel et al. 2018) and SPHERE NIR images (Avenhaus et al. 2018), and is consistent with the relatively high stellar rotation rate of $\sim 29 \mathrm{~km} \mathrm{~s}^{-1}$ (cf. Frasca et al. 2017).

The U-excess continuum emission in MY Lup is not evident (see Fig. E.8 in Alcalá et al. 2017) when using our methods (see Manara et al. 2013a; Alcalá et al. 2014, 2017) to derive the accretion luminosity, $L_{\text {acc}}$. Based on that analysis, which basically measures the Balmer continuum excess emission with respect to the photospheric one, including also the chromospheric level from a non-accreting template, we estimated an upper limit on $L_{\text {acc }}$ of $0.005 L_{\odot}$, which means an upper limit on $\dot{M}_{\text {acc }}<2.24 \times 10^{-10} M_{\odot} \mathrm{yr}^{-1}$, placing MYLup very close to the chromospheric noise level, and more than an order of magnitude below the typical $\dot{M}_{\text {acc }}$ value for YSOs of similar mass in the Lupus star forming region. We have also verified that the estimates of the limit on $L_{\text {acc }}$ derived from the Balmer continuum and the few emission lines detected are consistent (see Alcalá et al. 2017). According to the $\dot{M}_{\text {acc }}-\mathrm{WH} \alpha(10 \%)$ scatter plot for the Lupus sample shown in Fig. 9 of Alcalá et al. (2014), a WH $\alpha(10 \%) \sim 500 \mathrm{~km} \mathrm{~s}^{-1}$ measured in the UVES spectrum would suggest $\log \dot{M}_{\text {acc }} \approx-8.6$ for MYLup, but the spread of $\dot{M}_{\text {acc }}$ versus WH $\alpha(10 \%)$ is very large. Moreover, in the X-shooter spectrum used in Alcalá et al. (2017), the $\mathrm{H} \alpha$ line was not in emission as much as in the UVES and the other X-shooter spectrum (see Fig. 1). All this may lead to the conclusion that MY Lup is not accreting. This has been claimed to be the case for several transition discs, and in principle would be in line with the expectation from photoevaporation models (Ercolano et al. 2017; Ercolano \& Pascucci 2017, and references therein). However, it may be at odds with the detection of significant excess emission in the MIR and ALMA millimetre bands (see SED in Fig. 2 of van der Marel et al. 2018, and in Fig. 1 of Andrews et al. 2018). From radiative transfer modelling, which accounts for optical depth and disc inclination, Miotello et al. (2017) find a total dust mass of $\sim 173 M_{\text {Earth }}$.

\section{HST data}

\subsection{Observations and data processing}

The HST data used in this paper were acquired with the Cosmic Origins Spectrograph (COS) and the Space Telescope Imaging Spectrograph (STIS) onboard the HST as part of Cycle 24 General Observer program (PID 14 604; PI: C.F. Manara). The data were acquired in September 8, 2017 (UT start 01:35:54;

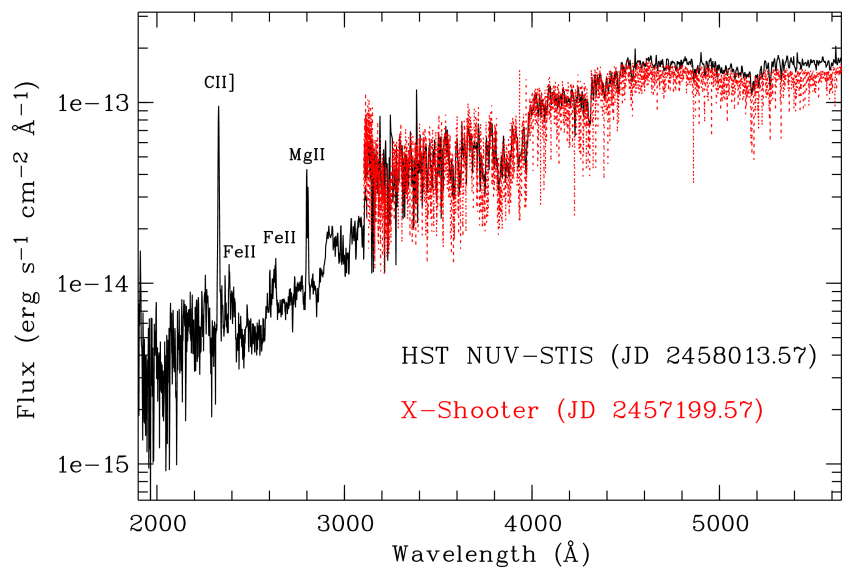

Fig. 2. Hubble Space Telescope NUV and STIS spectra of MY Lup are shown as the black continuous line. The red dotted line represents the $\mathrm{X}$-shooter spectrum acquired more than $2 \mathrm{yr}$ earlier as indicated by the JD in the labels. The spectra are corrected for reddening as described in the text.

UT end 10:07:17), which is more than two years after our X-shooter observation of June 26, 2015.

The data were collected in three different observing modes of HST-COS with the gratings G140L, G130M, and G160M, centred at $\lambda 1280, \lambda 1291$, and $\lambda 1577$, providing a resolving power $\sim 1500, \sim 16000$ and $\sim 16000$, respectively (see Green et al. 2012), as well as in two observing modes of HST-STIS using gratings G430L and G230L, centred at $\lambda 4300$ and $\lambda 2375$, respectively, both with a resolving power of $\sim 1000$ (see Woodgate et al. 1998, and references therein).

The G160M exposure time was 2592 s through the primary science aperture, with observations split between the 1577 and 1611 cenwave settings to mitigate fixed pattern noise and provide continuous spectral coverage over the $\mathrm{CIV}, \mathrm{H}_{2}$, and FUV continuum emitting wavelength regions. The velocity and flux calibration of the G160M spectra are $\pm 15 \mathrm{~km} \mathrm{~s}^{-1}$ and $5 \%$ absolute, respectively. The G130M and G140L spectra will be presented in a future work (Arulanantham et al., in prep.).

The data processing has been done in the same way as described in Arulanantham et al. (2018). Briefly, individual fluxcalibrated spectra generated by the COS pipeline are coadded using a cross-correlation algorithm (Danforth et al. 2010) that has been optimised for emission line sources (e.g. France et al. 2012). The data from different grating settings are then joined at the spectral overlap regions, with interpolated fluxes used to connect the observations from different gratings.

\subsection{Ultraviolet HST spectra}

Figure 2 shows the HST STIS spectra. Our X-shooter spectrum is overplotted as the red dotted line for comparison purposes. The spectra are corrected for reddening using $A_{\mathrm{V}}=1.3 \mathrm{mag}$ and adopting the extinction law by Cardelli et al. (1989) with $R_{\mathrm{V}}=3.1$ (however, see discussion on reddening in Calvet et al. 2004). We note that the fluxes of the HST-optical and X-shooter spectra are very consistent, despite being acquired more than $2 \mathrm{yr}$ apart. The very small slope decrease in the reddest spectral range can be explained by an extinction change $\Delta A_{\mathrm{V}}<0.2 \mathrm{mag}$. However, this is within the uncertainties of the $\mathrm{X}$-shooter analysis. The other X-shooter spectrum is also consistent with the HST data. Strong emission in the C II] $\lambda 2325$ and $\mathrm{Mg}$ II $\lambda 2800$ lines is also detected in the NUV spectrum. The latter line is related to accretion, but has also been observed in 


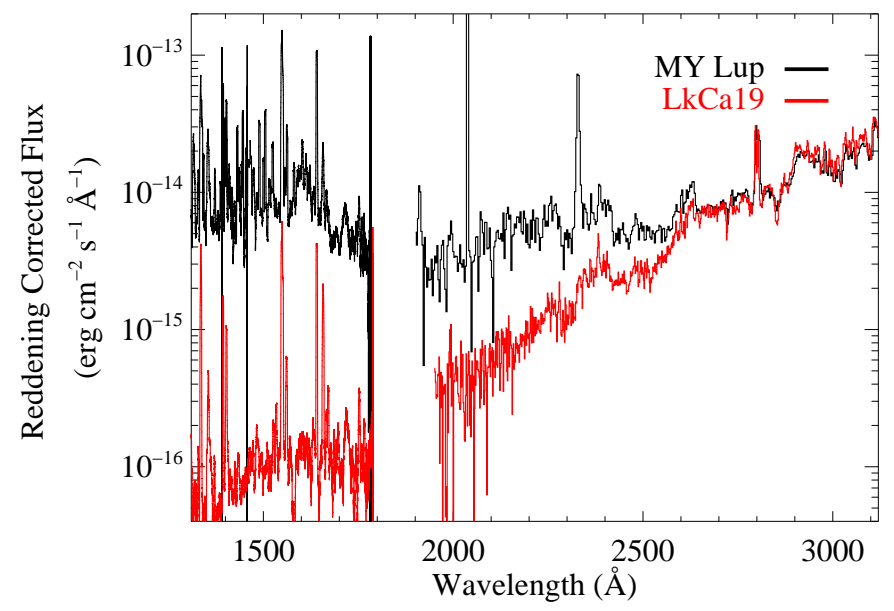

Fig. 3. Reddening corrected FUV spectrum, and part of the NUV spectrum, of MY Lup are shown with the black lines. The spectra of the $\mathrm{T}$ Tauri star LkCa 19 are overplotted with red lines. The FUV continuum, $\mathrm{H}_{2}$ fluorescence, and $1600 \AA$ Bump, characteristic of accreting objects (France et al. 2017), are observed in the spectrum of MY Lup.

non-accreting stars, while the former is much stronger in accretors than in non-accretors (Ingleby et al. 2011) and has been observed in other actively accreting sources (Calvet et al. 2004; Gómez de Castro \& Ferro-Fontán 2005; Ingleby et al. 2011). The tentative presence of the [O I] $\lambda 6300$ forbidden line may in principle lead to the idea that the semi-forbidden $\left.\mathrm{C}_{\mathrm{II}}\right] \lambda 2325$ line in MY Lup might form in a less dense environment than in the accretion flows, possibly in an outflow. However, only an upper limit for the flux of the [O I] line could be derived in Nisini et al. (2018). Moreover, the outflow interpretation for the C II] 12325 line is for stars that have strong outflows (Gómez de Castro \& Ferro-Fontán 2005). Therefore, the C II] $\lambda 2325$ emission line in MY Lup is mostly formed in the accretion flows.

In Fig. 3 the reddening corrected FUV spectrum, and part of the NUV spectrum, of MY Lup are shown. The spectra of the non-accreting YSO LkCa 19 are also overplotted as a red line for comparison. This star is also of K0-type, has a mass of $1.36 M_{\odot}$, similar within errors to MY Lup, and extinction $\mathrm{A}_{V}=0 \mathrm{mag}$ (see Kenyon \& Hartmann 1995; Kraus \& Hillenbrand 2009) $)^{1}$. Its projected rotational velocity was measured at $18.6 \mathrm{~km} \mathrm{~s}^{-1}$ (Hartmann et al. 1987) and a rotational period of 2.2 days has been reported in Clarke \& Bouvier (2000). It is confirmed that there are no signs of accretion in this star and therefore it can be used as a good comparison template to asses the accretion activity in MY Lup. The spectra of LkCa 19 (Ardila et al. 2013) were smoothed to $\sim 3 \AA$ spectral resolution to be comparable to the MY Lup spectrum.

A significant UV continuum excess emission and many emission lines tracing the inner disc $\left(\mathrm{H}_{2}\right.$ and $\left.\mathrm{CO}\right)$ and star-disc interaction region ( $\mathrm{N} \mathrm{V}, \mathrm{C} I \mathrm{~V}$, etc.) are detected in MYLup. In this work, we focus on a few lines that allow us to investigate the accretion properties, in particular, the C IV $\lambda 1549$ doublet, and the $\mathrm{H}_{2} \lambda 1547$ and $\mathrm{C}$ II] $\lambda 2325$ lines. In a forthcoming paper (Arulanantham et al., in prep.), a more detailed analysis of the complete set of detected lines will be presented.

The FUV spectrum of MY Lup shows both the C IV $\lambda 1549$ doublet and dozens of $\mathrm{H}_{2}$ lines including the $\mathrm{H}_{2} \lambda 1547$ molecular emission (see Fig. 4). The $\mathrm{H}_{2}$ lines are normally ascribed to gasrich inner discs around accreting YSOs (see France et al. 2012;

1 We note that Herczeg \& Hillenbrand (2014) adopt also a low value of $A_{V}=0.5 \mathrm{mag}$.

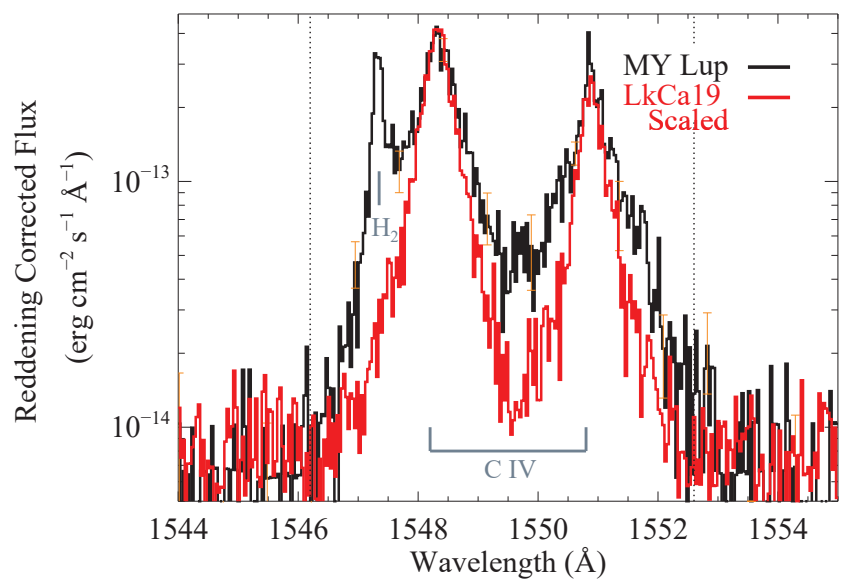

Fig. 4. Far-ultraviolet spectrum of MY Lup in the region of the $\mathrm{H}_{2}$ and C IV doublet is shown with the black continuous line. The spectrum of the non-accreting young star $\mathrm{LkCa} 19$ is overplotted as a red line.

Ingleby et al. 2011; Ardila et al. 2013; Hoadley et al. 2015, and references therein). $\mathrm{H}_{2}$ fluorescence is observed in all accreting protostars observed with HST to date, while no stars without dust discs displaying $\mathrm{H}_{2}$ emission have been discovered (France et al. 2012).

The spectrum of the non-accreting YSO LkCa 19 is also overplotted in Fig. 4 as a red line for comparison. This comparison shows that the profiles of the C IV $(\lambda \lambda 1548.2,1550.8)$ lines in MY Lup are broader than those of the non-accreting star, leading to the conclusion that MY Lup is accreting.

In summary, the presence of $\mathrm{H}_{2}$ lines, the higher level of FUV continuum flux with respect to that of the non-accreting star, and the presence of the strong C II] $\lambda 2325$ line, as well as the $\mathrm{H} \alpha$ profile and its variability, all strongly suggest that MY Lup is an actively accreting object.

\section{Analysis of HST-UV spectra}

\subsection{Ultraviolet continuum emission}

We can use the slab model derived for MYLup in Alcalá et al. (2017) and the spectrum of the non-accreting star to investigate by how much the UV excess emission is eventually underestimated.

The lower panel of Fig. 5 shows how the slab model derived from the X-shooter data in our previous analysis, plus the spectrum of the non-accreting star, fail to match the level of NUV continuum flux from MY Lup, albeit still with a relative good match in optical bands. At about $2000 \AA$ this model yields at least a factor seven less flux than observed, and as shown in the upper panel of the same figure, a slab at least a factor ten brighter would be required to achieve the continuum flux in the NUV. This would imply an accretion luminosity of more than an order of magnitude higher than our estimates based on X-shooter and is consistent with the results of the analysis of the C IV emission lines, as is described in the following sections. While in our previous analysis we used HBC 407 as a non-accreting template, also a K0-type star (Herczeg \& Hillenbrand 2014), the optical spectrum is also well fitted when using $\mathrm{LkCa} 19$ as a template.

As can be seen from the inset in Fig. 5, the X-shooter spectrum shows no significant Balmer jump, which may be reconciled by fitting the spectrum with an almost optically thick slab. However, the fit deviates from the photospheric features at short wavelengths, that is, the observed NUV spectrum is fainter 


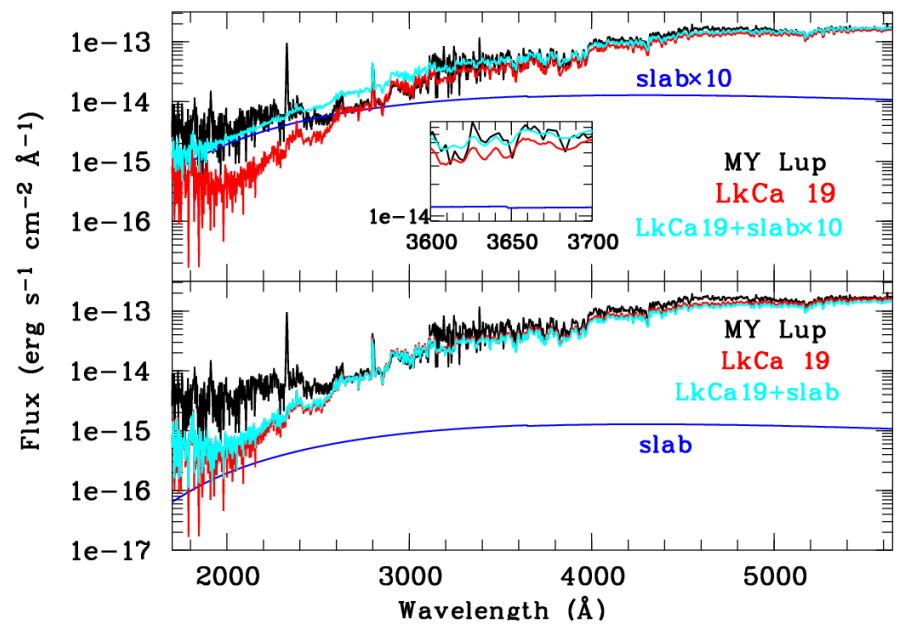

Fig. 5. Lower panel: extinction corrected HST NUV and STIS spectra of MY Lup (black) and LkCa 19 (red). The slab model derived in Alcalá et al. (2017) is also shown (blue). The cyan spectrum shows the sum of LkCa 19 plus the slab. Upper panel: same as in lower panel, but with the slab model multiplied by ten. We note the tiny Balmer Jump shown in the inset.

than the slab fit over some wavelengths $(\sim 2400-3000 \AA)$. This may be because the fit is very sensitive to the depth of photospheric features and therefore to any mismatch between the spectral features of MY Lup and those of the templates.

\subsection{The CIV $\lambda 1549$ doublet}

The C IV $\lambda 1549$ line emission in YSOs has been shown to correlate with the accretion luminosity, and therefore with the mass accretion rate, indicating that it is most likely originated in the accretion shock and flows (Johns-Krull et al. 2000; Ingleby et al. 2011; Ardila et al. 2013; France et al. 2014). To quantify how much of the CIV $\lambda 1549$ flux in MYLup can be attributed to excess accretion emission, one should compare the fluxes to those of the non-accreting star, scaled to match the peak fluxes of the narrow components, because these are likely dominated by the magnetic activity in the transition region of the protostellar atmosphere.

A line decomposition procedure, fitting the broad and narrow components of the C IV $\lambda 1549$ doublet (see Fig. 6 and Table 1), yields a total extinction-corrected $\left(A_{\mathrm{V}}=1.3 \mathrm{mag}\right.$, Alcalá et al. 2017) flux of $6.0( \pm 0.3) \times 10^{-13} \mathrm{erg} \mathrm{s}^{-1} \mathrm{~cm}^{-2}$. Subtracting the scaled C IV flux $\left(f_{\text {Civ }}=4.6 \times 10^{-13} \mathrm{erg} \mathrm{s}^{-1} \mathrm{~cm}^{-2}\right)$ from the non-accreting young star $\mathrm{LkCa} 19$ from the total flux in MYLup we end up with an excess CIV flux of $1.4( \pm 0.3) \times 10^{-13} \mathrm{erg} \mathrm{s}^{-1} \mathrm{~cm}^{-2}$. The extinction corrected flux of the $\mathrm{H}_{2}$ line is estimated at $5.5( \pm 0.6) \times 10^{-14} \mathrm{erg} \mathrm{s}^{-1} \mathrm{~cm}^{-2}$. Likewise, the flux of the $\mathrm{C}$ II] $\lambda 2325$, measured from the extinction corrected HST NUV spectrum is $6.0( \pm 0.5) \times 10^{-13} \mathrm{erg} \mathrm{s}^{-1} \mathrm{~cm}^{-2}$.

The luminosity of the lines can be computed as $L_{\text {line }}=$ $4 \pi d^{2} \cdot f_{\text {line }}$, where $d=156 \mathrm{pc}$ is the Gaia DR2 distance (Gaia Collaboration 2018) and $f_{\text {line }}$ is the extinction-corrected flux. The total luminosity of the C IV lines is $4.1( \pm 0.2) \times 10^{-4} L_{\odot}$, while the excess luminosity of the line, ECIV, with respect to the non-accreting star is $9.5( \pm 0.2) \times 10^{-5} L_{\odot}$. The luminosity of the $\mathrm{H}_{2}$ line alone is $3.8( \pm 0.4) \times 10^{-5} L_{\odot}$, and that of the C II] $\lambda 2325$ line is $4.1( \pm 0.4) \times 10^{-4} L_{\odot}$.

Ardila et al. (2013) demonstrated that the C IV line shape parameters (the velocity of the peak of the lines and width)

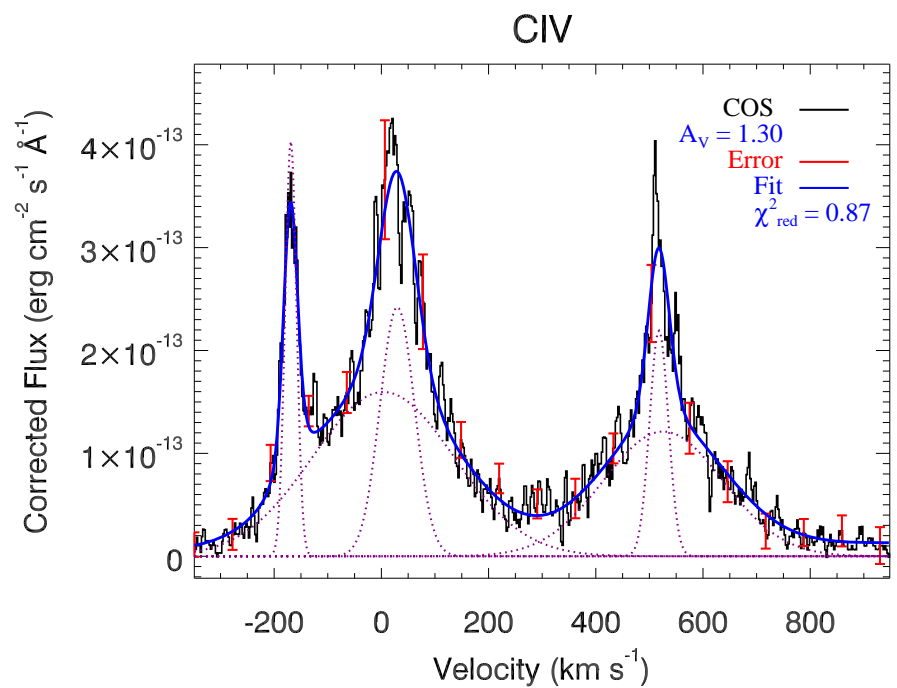

Fig. 6. Line decomposition fits of the CIV $\lambda 1549$ doublet and the $\mathrm{H}_{2}$ line. The extinction corrected $\left(A_{\mathrm{V}}=1.3 \mathrm{mag}\right) \mathrm{COS}$ spectrum is shown with the black line, whereas the fit is shown with the blue continuous line. The individual broad and narrow components are shown as the dotted lines.

Table 1. Output parameters of the fits to the $\mathrm{C} I V$ and $\mathrm{H}_{2}$ lines.

\begin{tabular}{lrcr}
\hline \hline Line ${ }^{\star \star}$ & $\begin{array}{r}V_{\text {cen }}( \pm \text { err }) \\
\left(\mathrm{km} \mathrm{s}^{-1}\right)\end{array}$ & $\begin{array}{c}\text { Flux }( \pm \text { err }) \\
\left(\times 10^{-14} \mathrm{erg} \mathrm{s}^{-1} \mathrm{~cm}^{-2}\right)\end{array}$ & $\begin{array}{r}F W H M( \pm \text { err }) \\
\left(\mathrm{km} \mathrm{s}^{-1}\right)\end{array}$ \\
\hline $\mathrm{H}_{2}$ & $-168.9(0.7)$ & $5.5(0.6)$ & $24.9(2.1)$ \\
$\mathrm{C}$ IV $(1548)-\mathrm{N}$ & $29.7(1.7)$ & $10.1(0.9)$ & $75.8(5.1)$ \\
C IV(1548)-B & $3.7(4.1)$ & $27.2(1.8)$ & $309.6(16.5)$ \\
C IV(1550)-N & $517.9(1.4)$ & $5.0(0.6)$ & $41.1(4.0)$ \\
C IV $(1550)-\mathrm{B}$ & $525.7(2.6)$ & $17.5(1.0)$ & $262.2(9.7)$ \\
\hline
\end{tabular}

Notes. ${ }^{(\star)}$ "N" and "B" stand for narrow and broad component, respectively.

do not correlate with accretion rate, but also that all accreting stars should show a broad component. The analysis of these latter authors also suggests that the accretion process first generates the broad component, with the narrow component becoming increasingly important at larger accretion rates. For low accretion rates $\left(<4 \times 10^{-9} M_{\odot} \mathrm{yr}^{-1}\right)$, the average narrow-component contribution to the luminosity is about $20 \%$, while for high accretion rates it is about $40 \%$ on average. The decomposition procedure of the C IV lines in MY Lup provides velocities and FWHM for the components (Table 1) that are more consistent with those of accreting objects than with those of non-accreting stars. Also, the scale factor from the $1550 \AA$ to the $1548 \AA \mathrm{C}$ IV line of MY Lup is 1.45 , as in many accreting objects. The contribution of the narrow components in MY Lup to the total luminosity is $25 \%$, that is, just above the limit for low accretors and is consistent with the narrow component contribution in other accreting objects with similar accretion rates to those of AA Tau and IP Tau (see Fig. 8 in Ardila et al. 2013). This suggests that the C IV doublet in MY Lup is produced by accretion.

\subsection{Accretion properties}

According to the $L_{\mathrm{C} \text { iv }}$ versus $L_{\text {acc }}$ relationships by Ingleby et al. (2011, their Fig. 10) and Yang et al. (2012) the C IV luminosity 
of $4.1 \times 10^{-4} L_{\odot}$ in MY Lup would imply an accretion luminosity of $\sim 2 \times 10^{-1} L_{\odot}$, that is, more than an order of magnitude higher than the upper limit derived from the optical data. We note that the $L(\mathrm{C} \mathrm{II}])$ versus $L_{\text {acc }}$ relationship derived by Ingleby et al. (2013, their Eq. (15)) and the C II] $\lambda 2325$ luminosity estimated in the previous section yield $L_{\mathrm{acc}}=1 \times 10^{-1} L_{\odot}$. Such $L_{\text {acc }}$ values would be consistent with those of accreting YSOs in Lupus and yield a mass accretion rate $\dot{M}_{\text {acc }} \sim(0.5-1) \times 10^{-8} M_{\odot} \mathrm{yr}^{-1}$. The latter calculated as

$\dot{M}_{\mathrm{acc}}=\left(1-\frac{R_{\star}}{R_{\mathrm{in}}}\right)^{-1} \frac{L_{\mathrm{acc}} R_{\star}}{G M_{\star}} \approx 1.25 \frac{L_{\mathrm{acc}} R_{\star}}{G M_{\star}}$,

assuming $\frac{R_{\star}}{R_{\text {in }}}=\frac{1}{5}$, where $R_{\star}$ and $R_{\text {in }}$ are the YSO radius and inner-disc radius, respectively (see Gullbring et al. 1998; Hartmann 1998), and using the stellar parameters reported in Alcalá et al. (2017). A similar value of $\dot{M}_{\text {acc }}=8 \times 10^{-9} M_{\odot} \mathrm{yr}^{-1}$ is derived when using the relationship between C IV luminosity and mass accretion rate provided by Ardila et al. (2013), that is, $\log \dot{M}_{\text {acc }}=0.8 \times \log L_{\mathrm{Civ}}-5.4$.

It is important to stress that the relationships between CIV-luminosity and accretion properties show a significant scatter, mainly because of a combination of non-simultaneity and extinction errors. Considering the total CIV luminosity, and taking into account the scatter in the Ingleby et al. (2011) and Yang et al. (2012) relationships, we estimate the accretion luminosity to be in the range $(0.9-4.0) \times 10^{-1} L_{\odot}$, which gives an accretion rate in the range $(0.4-1.8) \times$ $10^{-8} M_{\odot} \mathrm{yr}^{-1}$, implying a range of about $0.65 \mathrm{dex}$ in $\log \dot{M}_{\mathrm{acc}}$. Similarly, when using the Ardila et al. (2013) relationship and taking into account uncertainties in the component subtraction procedure and extinction, we estimate the accretion rate to be in the range $(0.5-2.5) \times 10^{-8} M_{\odot} \mathrm{yr}^{-1}$, meaning a range of about 0.7 dex in $\log \dot{M}_{\text {acc }}$. Although Ardila et al. (2013) find no significant difference when using all their data or only simultaneous data to derive their $\dot{M}_{\text {acc }}-L_{\mathrm{C} \text { iv }}$ relationship, they conclude that the exact relationship remains uncertain.

Using Eq. (2) by Johns-Krull et al. (2000), which considers correction for the presence of emission from the transition region, and the excess luminosity EC IV calculated in the previous section, we estimate a mass accretion rate of $2.4 \times$ $10^{-8} M_{\odot} \mathrm{yr}^{-1}$, that is, a value in the high end of the ranges quoted above. Considering errors and the scatter of the relationships, the $\dot{M}_{\text {acc }}$ values are in fair agreement. The derived $\dot{M}_{\text {acc }}$ for MY Lup covers a range of values accounting for the spread in possible contribution of the transition region.

Another important source of error is represented by the uncertainty in extinction, which in the FUV is a factor of about 2.6 higher than in the optical. We estimated an uncertainty in $A_{\mathrm{V}}$ of about 0.4-0.5 mag (Alcalá et al. 2014, 2017), which translates to a $\sim 0.4$ dex error in $\log L_{\mathrm{Civ}}$. According to the $L_{\mathrm{C} \text { iv }}$ versus $L_{\text {acc }}$ relationships used above, this implies $L_{\text {acc }}$ in the range $(0.7-$ $4.0) \times 10^{-1} L_{\odot}$. Thus, the range of $\dot{M}_{\text {acc }}$ due to the effect of the uncertainty in $\mathrm{A}_{\mathrm{V}}$ is estimated to be $(0.3-1.8) \times 10^{-8} M_{\odot} \mathrm{yr}^{-1}$.

We conclude that a reasonable mass accretion rate for MY Lup is $\dot{M}_{\text {acc }} \sim 1\left({ }_{-0.5}^{+1.5}\right) \times 10^{-8} M_{\odot} \mathrm{yr}^{-1}$. Beyond the relatively large uncertainty in $\dot{M}_{\text {acc }}$, the luminosity of the C IV lines suggests similar $\dot{M}_{\text {acc }}$ values as those of bona fide accreting YSOs with comparable mass. This can be seen in Fig. A.1, where the stellar mass and accretion rates of the Lupus sample are revisited using the distances drawn from Gaia DR2. It is worth noting that the updated value for the mass accretion rate is also consistent with the more luminous slab model described in Sect. 4.1.

\section{Discussion}

\subsection{The $X$-shooter-COS-STIS discrepancy on $L_{a c c}$}

Among the several objects where HST and X-shooter data exist, MYLup is the only star for which a discrepancy has been observed so far. In fact, the several HST-based $\dot{M}_{\text {acc }}$ values found by Ingleby et al. (2013) and those found by Manara et al. (2014) are in good agreement. Also, an additional data set of the same observing program from which the MY Lup HST data are taken show good agreement between the HST and X-shooter $\dot{M}_{\text {acc }}$ values for several stars (Arulanantham et al., in prep.). Therefore, MY Lup seems to be the only real outlier so far. The emission lines, and substantial FUV excess emission detected in the HST spectra of MY Lup strongly suggest that the star is an actively accreting object, whereas the X-shooter data show weak or no accretion. MY Lup is peculiar in several respects. Besides being one of the most massive $\mathrm{T}$ Tauri stars in Lupus, it is an almost edge-on disc. Hence, both the effects of low contrast in photospheric versus continuum excess emission and the occultation of some accretion diagnostics may play different roles in the $\mathrm{X}$-shooter accretion diagnostics and in those of HST. In principle, the accretion flows might be obscured by the highly inclined disc hampering the detection of excess emission in the X-shooter wavelength range, but such an effect would be much stronger for the C IV lines. We note however that Ardila et al. (2013) find no correlation between inclination and the C IV luminosity of accretion rate, hence conclude that there is no evidence that the disc or the accretion flow are obscuring the accretion diagnostics. In their interpretation, the C IV UV lines are not emitted from a particular place, but cover the whole star. On one hand, very weak or no accretion would imply very little gas content in the disc, consistent with the finding of a rather low gas-todust mass ratio from faint CO isotopologue ALMA observations by Miotello et al. (2017). Nonetheless, we highlight the fact that these authors also discuss the possibility of a normal gas-to-dust ratio but a disc highly depleted in $\mathrm{CO}$, yielding artificially low gas mass estimates. On the other hand, the HST-UV tracers seem to point toward accretion more typical of Class II discs and of some transitional discs in Lupus and Chamaeleon I (Alcalá et al. 2017; Manara et al. 2017a).

As in previous studies (cf. Calvet et al. 2004; Ingleby et al. 2011; Manara et al. 2014), this further confirms that FUV measurements represent a reliable way to establish the presence of accretion in low-accreting, early-type, intermediate-mass T Tauri stars. It is also worth stressing that the width of the $\mathrm{H} \alpha$ line in this type of object may already suggest active accretion (Romero et al. 2012 and see also the case of SR 21 in Manara et al. 2014). The complex $\mathrm{H} \alpha$ profile, which is consistent with the model prediction by Kurosawa et al. (2006, see their Fig. 13) of a highinclination angle, also supports the conclusion of significant UV excess emission due to accretion, and of a complex geometry of the infalling streams.

\subsubsection{Possible stellar flare?}

One question that may arise is whether some of the emission lines observed in the COS data, in particular the $\mathrm{H}_{2}$ lines, could be produced by a strong flare and not by accretion. As discussed in Hoadley et al. (2015) and Arulanantham et al. (2018, and references therein), the $\mathrm{H}_{2}$ lines are formed by fluorescence of Ly- $\alpha$ photons. In low-mass stars, just the chromospheric Ly- $\alpha$ emission alone can generate detectable $\mathrm{H}_{2}$ fluorescence from the stellar atmosphere (Kruczek et al. 2017). However, a much more 


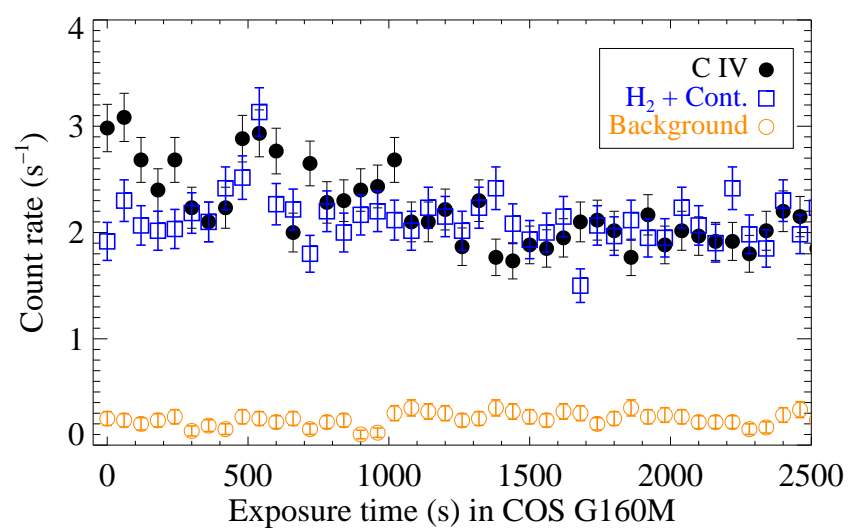

Fig. 7. Far-ultraviolet light curves for a few relevant lines related to a possible flare and Ly- $\alpha$-driven fluorescence. The $\mathrm{C}$ IV region was integrated over $1544-1556 \AA$ and the $\mathrm{H}_{2}$ region was integrated over $1488-1526 \AA$. The total integration time was $2592 \mathrm{~s}$ and the temporal cadence in the plots is $60 \mathrm{~s}$ per time step.

intense Ly- $\alpha$ pumping would be needed to explain the bright $\mathrm{H}_{2}$ emission that we see at the distance of $156 \mathrm{pc}$ of MY Lup. In the Sun and cool dwarfs, the $\mathrm{H}_{2}$ lines are produced by narrow Ly-alpha, while accretors have broad Ly- $\alpha$ lines.

One argument against the flare scenario is that the COS G130M and G160M data were not acquired simultaneously; both show the same level of $\mathrm{H}_{2}$ emission, as do the G140L data. Furthermore, COS data is acquired in time-tag mode and light curves can be created as a function of wavelength. Figure 7 shows light curves for the $\mathrm{H}_{2}$ and $\mathrm{C}$ IV lines. The light curves of the G160M data did not show any signs of significant temporal variability. Therefore, we conclude that the emission lines observed in the COS data are not related to flare events, but that active accretion is the explanation of the UV emission.

\subsubsection{Variability}

MYLup is a variable star by definition. Time series BVRI photometry gathered from the AAVSOnet Epoch Photometry Database $^{2}$ shows that the maximum amplitude of variations in the $V$-band is on the order of $0.6 \mathrm{mag}$, with the tendency for the source to become redder when fainter. The latter is consistent with a variable circumstellar extinction due to the edge-on geometry. We note however that the long-term light curves from the Digital Access to a Sky Century at Harvard (DASCH) ${ }^{3}$ suggest that events of up to $1.5 \mathrm{mag}$ in extinction may occur in MY Lup. In any case, the comparison in Fig. 2 shows that the flux of the $\mathrm{X}$-shooter spectrum and the STIS spectrum are not significantly different, despite being acquired more than $2 \mathrm{yr}$ apart. The very small slope difference in the reddest spectral range can indeed be explained by an extinction change $\Delta A_{\mathrm{V}}<0.2 \mathrm{mag}$ observed in the AAVSOnet data. Such variability would not explain an order of magnitude difference in the $L_{\text {acc }}$ estimates.

The comparison in Fig. 2 does not exclude, however, that larger variations in the UV, mostly unnoticed in the optical and due to variable accretion, may occur in other epochs. MY Lup was detected by GALEX in the NUV only, with an AB magnitude of 17.49 mag. A standard IDL software that converts spectra directly into a GALEX AB magnitude applied to our STIS spectrum of MYLup yields an AB magnitude of $19.40 \mathrm{mag}$,

\footnotetext{
2 https://www .aavso.org/aavsonet-epoch-photometrydatabase

3 http://dasch.rc. fas.harvard.edu/lightcurve.php
}

suggesting a factor of 5-6 variability in the NUV flux, presumably related to accretion luminosity variability and/or inner disc occultation. Variability by factors of approximately five in FUV flux from accreting YSOs have previously been detected with HST, and these tend to be sources with variable disc attenuation like AA Tau, RW Aur, and T Cha (see for instance Schneider et al. 2015, for AA Tau). High variability has also been observed in multi-epoch UV HST observations of accreting lowmass stars (Robinson \& Espaillat 2019). Thus, the comparison with GALEX shows that variations in the UV in MY Lup are in line with those observed in other accreting low-mass stars. Objects like FUOrs and EXOrs may exhibit much higher levels of variability, but most of these objects are more embedded and younger than $\mathrm{T}$ Tauri stars, and in the above-mentioned photometric data of MY Lup there is no evidence for a FUOri/EXLup type behaviour. We also stress that, except for the changes seen in the $\mathrm{H} \alpha$ profile (see Fig. 1), no significant variability is observed among the two X-shooter spectra available so far, either in flux or slope of the spectra.

\subsubsection{Anomalous extinction}

Other relevant effects that may have an important impact on the flux measurements of the continuum and lines at different wavelengths are represented by a possible deviation from a normal extinction law. Regarding anomalous extinction, and especially for high-inclination objects, if one exchanged part of the absorbing material that has $R_{\mathrm{V}}=3.1$ with material that has for example $R_{\mathrm{V}}=4.0$, this could lead to an underestimation of the C IV fluxes because the FUV reddening correction is smaller for higher $R_{\mathrm{V}}$. However, this change might go unnoticed in the optical when only part of the absorber is effected. Recent SPHERE observations in scattered light provide evidence of spatial segregation of dust grains and different (small/large) grain population and distributions in SST c2dJ160830.7-382827 (Villenave et al. 2019). This object is the other transitional disc found to show weak or no accretion based on the X-shooter data (see Sect. 1) and is very similar in many respects to MY Lup. However, even assuming $R_{\mathrm{V}}=5.5$ would reduce the value of the extinction-corrected flux of the C IV lines by a factor of about four, but the resulting $\dot{M}_{\text {acc }}$ would still be higher than the X-shooter estimate by about 0.8 dex in $\log \dot{M}_{\text {acc }}$.

\subsubsection{Other effects}

The effects of scattering in the FUV may also be important. For instance, repeated observations of the T Tauri star AA Tau have shown that the C IV fluxes did not decrease as much as expected during the dim state (see details in Schneider et al. 2015).

\subsubsection{Excess emission in the FUV}

Previous works (Calvet et al. 2004; Ingleby et al. 2011; Manara et al. 2014) have pointed out that the excess emission for intermediate-mass stars is hard to detect at wavelengths longer than $3000 \AA$ because the temperatures of the stellar photosphere and the regions of the photosphere heated by the accretion are similar. MY Lup is one of the hottest (K0-type) T Tauri stars in Lupus, and therefore the contrast between continuum excess emission with respect to the photospheric+chromospheric emission, which reduces in the objects with spectral type earlier than about K3, may hamper detection of excess emission in the X-shooter wavelength range. The excess FUV continuum emission in MYLup is evident, and is present in almost all accreting sources, as shown in France et al. (2017). This latter 
study of a sample of accreting $\mathrm{T}$ Tauri stars showed a strong correlation between the FUV continuum luminosity and the total luminosity of the C IV lines (see their Fig. 6) ${ }^{4}$. We estimated the reddening-corrected FUV continuum luminosity in MY Lup to be $\sim 3.6 \times 10^{30} \mathrm{erg} \mathrm{s}^{-1}$, while the total luminosity of the C IV lines is $\sim 1.6 \times 10^{30} \mathrm{erg} \mathrm{s}^{-1}$, which places MY Lup in very good agreement on that correlation. In addition, the " $1600 \AA$ Bump" observed in almost all the accreting objects investigated in Ingleby et al. (2009), France et al. (2017), and Espaillat et al. (2019), is also present in MY Lup (see Fig. 3), further putting it in the class of gas-rich, accreting objects.

\subsection{The MYLup disc}

The age estimate of $\sim 17$ Myr for MYLup (Alcalá et al. 2017; Frasca et al. 2017) is much higher than the average for the Lupus members, which is in principle consistent with a more evolved disc. Baraffe \& Chabrier (2010) concluded that episodic strong accretion during PMS evolution of low-mass stars may produce objects with smaller radius, higher central temperature, and lower luminosity compared to the non-accreting counterparts of the same mass and age, resulting in low-luminosity objects. Also, Kunimoto et al. (2017) and Baraffe et al. (2017) conclude that accretion history may affect PMS evolution leading to a luminosity spread in the HR diagram. The age of MY Lup may however be overestimated because the highly inclined disc partially obscures the stellar photosphere. The star may appear underluminous on the HR diagram if the entire stellar surface is not subject to the same extinction and part of it is almost invisible. This effect, possibly due to a warped inner dusty disc, has been observed in other objects like V 354 Mon (see Schneider et al. 2018). Thus, MY Lup may indeed be much younger than what its position on the HR diagram would suggest. From the gravity $\log g=3.72 \pm 0.18$ derived by Frasca et al. (2017), and assuming the mass of $1.1 M_{\odot}$, we estimate a radius of $2.2 R_{\odot}$ for the star, and therefore a luminosity of $3.14 L_{\odot}$, which is a factor of about four higher, placing MY Lup on the HR diagram in good agreement with the other Lupus members. This would imply a $\dot{M}_{\text {acc }}=1.6 \times 10^{-8} M_{\odot} \mathrm{yr}^{-1}$. This $\dot{M}_{\text {acc }}$ value estimated from the HST-UV data is indeed similar to the average derived for Lupus Class II discs. The fact that MY Lup shows up as a Class II disc for the gas properties is in line with other results from X-shooter (e.g. Manara et al. 2014) and HST observations (see the case of RY Lup in Arulanantham et al. 2018, but also other objects in Hoadley et al. 2015), and in many cases the gas is clearly present in the large dust cavities detected by ALMA (Miotello et al. 2017; van der Marel et al. 2018, and references therein). We also note that recent studies (Manara et al. 2014, 2017b; Alcalá et al. 2014, 2017; van der Marel et al. 2018) find no substantial differences among the stellar and accretion properties of both primordial and transition discs (but see also Najita et al. 2007, 2015, for a different result), with the exception that the dusty transition discs with large cavities $\left(r_{\text {cav }}>15 \mathrm{AU}\right)$ in Lupus tend to be more massive than the Class II disc systems (van der Marel et al. 2018).

Combined studies of SEDs and millimetre data (Owen \& Clarke 2012; Ercolano \& Pascucci 2017) suggested two populations of transition discs: those with low disc masses and low or non-detectable accretion, in which the inner cavities are mainly due to photoevaporation, and those with large disc masses and high accretion rates, where the inner cavities are most likely created by giant planet formation. Also, the van der Marel

4 We note that this is the total integrated luminosity of the CIV lines, not only the one corresponding to the excess emission with respect to the non-accreting star. et al. (2018) study suggested two different evolutionary paths for the evolution of protoplanetary discs: small, low-mass discs which dissipate slowly without forming large dust cavities, and massive, large discs that go through a transition phase of disc clearing by giant planets.

The analysis by van der Marel et al. (2018) suggested a large inner dust cavity of $25 \mathrm{AU}$ in the MYLup disc. However, the continuum visibilities in this study show only a tentative cavity at best, and the DSHARP results of Huang et al. (2018) show a rather centrally peaked continuum emission. Therefore, a particularly deep and large dust cavity in the inner disc region is not evident, casting doubts on the classification as a TD. Moreover, given the high mass accretion rate of $1 \times 10^{-8} M_{\odot} \mathrm{yr}^{-1}$ measured from the UV-HST data, a large dusty cavity would not be easily explained in terms of photoevaporation models (see Fig. 9 in van der Marel et al. 2018). Some X-ray photoevaporation models might explain large cavities of accreting YSOs by imposing very low $\mathrm{C}$ and $\mathrm{O}$ disc abundances (Ercolano et al. 2017). This would be in principle consistent with the Miotello et al. (2017) scenario of a disc highly depleted in $\mathrm{CO}$, artificially leading to low gas-mass estimates. Yet, the estimated $\log \dot{M}_{\text {acc }}$ value of approximately -8.0 for MYLup is still high to be compatible with those models. Therefore, if there is an inner cavity in the disc of MY Lup, it may have been caused by giant planet formation.

We note that the SPHERE observations by Avenhaus et al. (2018) detected a ring in scattered light at a radius of about $120 \mathrm{AU}$ from the star. Also, the annular substructures observed by DSHARP (Huang et al. 2018) are possible features reproduced in simulations of planet-disc interactions. Nevertheless, annular structures may not necessarily be the product of planetdisc interactions (see introduction in Huang et al. 2018, for other possibilities). Analysis of the ALMA and SPHERE data of MYLup by C. Walsh (priv. comm.) shows that the disc is rather flared, and the gaps and rings seen in the sub-millimetre DSHARP data are relatively shallow. Further detailed radiative transfer modelling of the disc using the data gathered within DSHARP (Andrews et al. 2018), as well as future direct imaging and observations at higher angular and spectral resolution of molecular lines, will provide additional information for determining the origin of the disc substructures in MY Lup.

\section{Conclusions}

We report new HST-COS and -STIS observations of MY Lup, found to be a very weak or even negligible accretor based on previous X-shooter observations. The UV-HST spectra show many emission lines, as well as strong continuum excess emission in the FUV, ascribable to accretion. This confirms that MY Lup is actively accreting mass at a rate of $\dot{M}_{\text {acc }}=1\left({ }_{-0.5}^{+1.5}\right) \times 10^{-8} M_{\odot} \mathrm{yr}^{-1}$, that is, a value similar to the average derived for Lupus Class II discs, and more than an order of magnitude above the previous X-shooter estimate. MYLup is the only case so far for which a significant difference between the HST and X-shooter $\dot{M}_{\text {acc }}$ estimates exists that is not ascribable to variability. Among the several possible reasons for the X-shooter-HST discrepancy are anomalous extinction and effects of scattering in the FUV. Indeed, MY Lup is peculiar in many respects: it is an almost edge-on disc and one of the hottest (K0-type) $\mathrm{T}$ Tauri stars in Lupus. Hence, detection of excess emission in the $\mathrm{X}$-shooter wavelength range may be hampered both by occultation effects from optically thick disc material along the line of sight and by the low contrast between continuum excess and photospheric+chromospheric emission expected for earlytype $(<\mathrm{K} 3)$ objects. The high FUV continuum luminosity of 
$\sim 3.6 \times 10^{30} \mathrm{erg} \mathrm{s}^{-1}$, and the total luminosity of the C IV lines of $\sim 1.6 \times 10^{30} \mathrm{erg} \mathrm{s}^{-1}$, as well as the strong $\mathrm{H}_{2}$ fluorescence and $1600 \AA$ Bump, place MY Lup in the class of gas-rich accreting objects. The mass accretion rate and a previously suggested large cavity of the dusty disc cannot be easily explained in terms of photoevaporation models. In fact, reviewing all available data suggests that no compelling evidence for a large dust cavity exists and we conclude that giant planet formation is simply a plausible scenario for the dust rings in the disc of MY Lup.

Acknowledgements. We thank the referee, Greg Herczeg, for his very useful and constructive comments which helped to improve a previous version of the paper. We thank Catherine Walsh and Nieke van der Marel for their very useful comments and discussions on the disc of MYLup, and Antonio Frasca for help and discussion on the photospheric spectrum subtraction. This work has been supported by the project PRIN-INAF 2016 "The Cradle of Life GENESIS-SKA (General Conditions in Early Planetary Systems for the rise of life with SKA)" and the PRIN-INAF-MAIN-STREAM 2017 "Protoplanetary disks seen through the eyes of new-generation instruments". This research made use of the SIMBAD database, operated at the CDS (Strasbourg, France) C.F.M. and A.M. acknowledge an ESO Fellowship. P.C.S. acknowledges support from the Deutsches Zentrum für Luft- und Raumfahrt through project 50OR1706. H.M.G. was supported by program HST-GO-15 204.001, which was provided by NASA through a grant from the Space Telescope Science Institute, which is operated by the Associations of Universities for Research in Astronomy, Incorporated, under NASA contract NAS5-26555. This work has made use of data from the European Space Agency (ESA) mission Gaia https: // www . cosmos . esa. int/gaia, processed by the Gaia Data Processing and Analysis Consortium (DPAC, https://www.cosmos.esa.int/web/gaia/dpac/ consortium). Funding for the DPAC has been provided by national institutions, in particular the institutions participating in the Gaia Multilateral Agreement. This project has received funding from the European Union's Horizon 2020 research and innovation programme under the Marie Skłodowska-Curie grant agreement No 823823 (DUSTBUSTERS). We acknowledge with thanks the variable star observations from the AAVSO International Database contributed by observers worldwide and used in this research. We also acknowledge the use of DASCH data: the DASCH project at Harvard is grateful for partial support from NSF grants AST-0407380, AST-0909073, and AST-1 313 370. This work was partly supported by the Deutsche Forschungs-Gemeinschaft (DFG, German Research Foundation) - Ref no. FOR 2634/1 TE 1024/1-1, by the DFG cluster of excellence Origin and Structure of the Universe (www . universe-cluster . de)

\section{References}

Alcalá, J. M., Natta, A., Manara, C., et al. 2014, A\&A, 561, A2 Alcalá, J. M., Manara, C., Natta, A., et al. 2017, A\&A, 600, A20

Alexander, R. D., Pascucci, I., Andrews, S., Armitage, P., \& Cieza, L. 2014, in Protostars and Planets VI, eds. H. Beuther, R. S. Klessen, C. P. Dullemond, $\&$ T. Henning (Tucson, AZ: University of Arizona Press), 475

Andrews, S. M., Wilner, D. J., Espaillat, C., et al. 2011, ApJ, 732, 42

Andrews, S. M., Huang, J., Pérez, L. M., et al. 2018, ApJ, 869, 41

Ansdell, M., Williams, J. P., van der Marel, N., et al. 2016, ApJ, 828, 46

Ardila, D. R., Herczeg, G. J., Gregory, S. G., et al. 2013, ApJS, 207, 1

Arulanantham, N. 2018, Take a Closer Look, 15-19 October, 2018 in ESO-

HQ, Garching b. München, Germany https://zenodo.org/record/ 1488765\#.XG_3H]x7m50

Arulanantham, N., France, K., Hoadley, K., et al. 2018, ApJ, 855, 98

Avenhaus, H., Quanz, S. P., Garufi, A., et al. 2018, ApJ, 863, 44

Baraffe, I., \& Chabrier, G. 2010, A\&A, 521, A44

Baraffe, I., Homeier, D., Allard, F., \& Chabrier, G. 2015, A\&A, 577, A42

Baraffe, I., Elbakyan, V. G., Vorobyov, E. I., \& Chabrier, G. 2017, A\&A, 597, A19

Batalha, C. C., Quast, G. R., Torres, C. A. O., et al. 1998, A\&A, 128, 561

Biazzo, K., Alcalá, J. M., Covino, E., et al. 2012, A\&A, 547, A104

Bustamante, I., Merín, B., Ribas, Á., et al. 2015, A\&A, 578, A23

Calvet, N., Muzerolle, J., Brice no, C., et al. 2004, AJ, 128, 129

Cardelli, J. A., Clayton, G. C., \& Mathis, J. S. 1989, ApJ, 345, 245

Cieza, L. A., Schreiber, M. R., Romero, G. A., et al. 2010, ApJ, 712, 925

Clarke, C. J., \& Bouvier, J. 2000, MNRAS, 319, 457

Comerón, F. 2008, Handbook of Star Forming Regions: The Southern Sky, Volume II, ed. B. Reipurth (San Francisco: ASP Monograph Publications), 5, 295
Costigan, G., Scholz, A., Stelzer, B., et al. 2012, MNRAS, 427, 1344 Costigan, G., Vink, J. S., Jorick, S., et al. 2014, MNRAS, 440, 3444 Danforth, Ch. W., Keeney, B. A., Stocke, J. T., Shull, J. M., \& Yao, Y. 2010, ApJ, 720,976

Ercolano, B., \& Pascucci, I. 2017, RSOS, 470114

Ercolano, B., Weber, M. L., \& Owen, J. E. 2017, MNRAS, 473, 64 Espaillat, C., Muzerolle, J., Hernández, J., et al. 2008, ApJ, 689, 145 Espaillat, C., Robinson, C., Grant, S., \& Reynolds, M. 2019, ApJ, 876, 121 France, K., Yang, H., \& Linsky, J. L. 2011, ApJ, 729, 7

France, K., Schindhelm, E., Herczeg, G. J., et al. 2012, ApJ, 756, 171

France, K., Herczeg, G. J., McJunkin, M., \& Penton, S. V. 2014, ApJ, 794, 160

France, K., Roueff, E., \& Abgrall, H. 2017, ApJ, 844, 169

Frasca, A., Biazzo, K., Alcalá, J. M., et al. 2017, A\&A, 602, A33

Gaia Collaboration (Brown, A. G. A., et al.) 2018, A\&A, 616, A1

Gómez de Castro, A. I., \& Ferro-Fontán, C. 2005, MNRAS, 362, 569

Green, J. C., Froning, C. S., Osterman, S., et al. 2012, ApJ, 744, 60

Gregorio-Hetem, J., Lepine, J. R. D., Quast, G. R., Torres, C. A. O., \& de La Reza, R. 1992, AJ, 103, 549

Gullbring, E., Hartmann, L., Brice no, C., \& Calvet, N. 1998, ApJ, 492, 323

Hartmann, L. 1998, Accretion Processes in Star Formation, (Cambridge: Cambridge University Press)

Hartmann, L. W., Soderblom, D. R., \& Stauffer, J. R. 1987, AJ, 93, 907

Hartmann, L. E., Calvet, N., Gullbring, E., \& D’Alessio, P. 1998, ApJ, 495, 385

Hartmann, L., Herczeg, G., \& Calvet, N. 2016, ARA\&A, 54, 135

Herczeg, G., \& Hillenbrand, L. A. 2008, ApJ, 681, 594

Herczeg, G., \& Hillenbrand, L. A. 2014, ApJ, 786, 97

Herczeg, G. J., Linsky, J. L., Valenti, J. A., Johns-Krull, C. M., \& Wood, B. E. 2002, ApJ, 572, 310

Herczeg, G. J., Wood, B. E., Linsky, J. L., Valenti, J. A., \& Johns-Krull, C. M. 2004, ApJ, 607, 369

Hoadley, K., France, K., Alexander, R. D., McJunkin, M., \& Schneider, P. C. 2015, ApJ, 812, 41

Huang, J., Andrews, S. M., Dullemond, C. P., et al. 2018, ApJ, 869, 42

Ingleby, L., Calvet, N., Bergin, E., et al. 2009, ApJ, 703, 137

Ingleby, L., Calvet, N., Bergin, E., et al. 2011, ApJ, 743, 105

Ingleby, L., Calvet, N., Herczeg, G., \& Briceño, C. 2012, ApJ, 752, 20

Ingleby, L., Calvet, N., Herczeg, G., et al. 2013, ApJ, 767, 1121

Johns-Krull, C. M., Valenti, J. A., Linsky, J. L., et al. 2000, ApJ, 539, 815

Kenyon, S., J., \& Hartmann, L. 1995, ApJS, 101, 117

Kraus, A. L., \& Hillendbrand, L. A. 2009, ApJ, 704, 547

Kruczek, N., France, K., Evonosky, W., et al. 2017, ApJ, 845, 3

Kunitomo, M., Guillot, T., Takeuchi, T., \& Ida, S. 2017, A\&A, 599, A49

Kurosawa, R., Harries, T. J., \& Symington, N. H. 2006, MNRAS, 370, 580

Manara, C. F., Beccari, G., Da Rio, N., et al. 2013a, A\&A, 558, A114

Manara, C. F., Testi, L., Rigliaco, E., et al. 2013b, A\&A, 551, A107

Manara, C. F., Testi, L., Natta, A., et al. 2014, A\&A, 568, A18

Manara, C. F., Fedele, D., Herczeg, G. J., \& Teixeira, P. S. 2016, A\&A, 585, A136

Manara, C. F., Frasca, A., Alcalá, J. M., et al. 2017a, A\&A, 605, A86

Manara, C. F., Testi, L., Herczeg, G. J., et al. 2017b, A\&A, 604, A127

Martin, D. C., Fanson, J., Schiminovich, D., et al. 2005, ApJ, 619, 1

Merín, B., Jørgensen, J. K., Spezzi, L., et al. 2008, ApJS, 177, 551

Merín, B., Brown, J. M., Oliveira, I., et al. 2010, ApJ, 718, 1200

Miotello, A., van Dishoeck, E. F., Williams, J. P., et al. 2017, A\&A, 599, A113

Morbidelli, A., \& Raymond, S. N. 2016, J. Geol. Resource Eng., 121, 1962

Morrissey, P., Schiminovich, D., Barlow, T. A., et al. 2005, ApJ, 619, 7

Najita, J. R., Strom, S. E., \& Muzerolle, J. 2007, MNRAS, 378, 369

Najita, J. R., Andrews, S. M., \& Muzerolle, J. 2015, MNRAS, 450, 3559

Nisini, B., Antoniucci, S., Alcalá, J. M., et al. 2018, A\&A, 609, A89

Owen, J. E., \& Clarke, C. J. 2012, MNRAS, 426, L96

Robinson, C., \& Espaillat, C. 2019, ApJ, 874, 129

Romero, G. A., Schreiber, M. R., Cieza, L. A., et al. 2012, ApJ, 749

Schindhelm, E., France, K., Burgh, E. B., et al. 2012, ApJ, 746, 97

Schneider, P. C., France, K., Günther, H. M., et al. 2015, A\&A, 584, A51

Schneider, P. C., Manara, C., Fachini, S., et al. 2018, A\&A, 614, A108

Siess, L., Dufour, E., \& Forestini, M. 2000, A\&A, 358, 593

Valenti, J. A., Johns-Krull, C. M., \& Linsky, J. L. 2000, ApJS, 129, 399

van der Marel, N., Williams, J. P., Ansdell, M., et al. 2018, ApJ, 854, 177

Venuti, L., Bouvier, J., Flaccomio, E., et al. 2014, A\&A, 570, A82

Vernet, J., Dekker, H., D'Odorico, S., et al. 2011, A\&A, 536, A105

Villenave, M., Benisty, M., dent, W. R. F., et al. 2019, A\&A, 624, A7

Vorobyov, E. I., \& Basu, S. 2009, ApJ, 703, 922

Woodgate, B. E., Kimble, R. A., Bowers, C. W., et al. 1998, PASP, 110, 1183

Yang, H., Herczeg, G. J., \& Linsky, J. L., et al. 2012, ApJ, 744, 121 


\section{Appendix A: Revisited stellar and accretion properties for the Lupus sample}

Table A.1 lists some of the stellar and accretion properties for the Lupus T Tauri stars in the Alcalá et al. (2017) sample as updated using the distances drawn form the Gaia DR2 (Gaia Collaboration 2018). The $M_{\star}$ and $\dot{M}_{\text {acc }}$ values are derived using both the Baraffe et al. (2015) and Siess et al. (2000) evolutionary tracks, and are respectively labelled as B15 and S00 in the table. The distances of the individual objects are not significantly different from those adopted in Alcalá et al. (2017) and therefore changes in the stellar and accretion properties are small. The main difference is that the YSOs in the Lupus III cloud are on the average at a distance of $159 \mathrm{pc}$ and not $200 \mathrm{pc}$ as previously assumed, while most of the YSOs in the other clouds are also at a distance of about $160 \mathrm{pc}$ and not $150 \mathrm{pc}$. There are three problematic objects namely Sz102, SSTc2dJ160703.9391112 and 2MASSJ16085373-3 914367 . The former is flagged with large parallax error, while the second has no measurement, and the third one has astrometric excess noise larger than 5. For these three we adopt the average value of $159 \mathrm{pc}$.

Figure A.1 shows the updated $\log \dot{M}_{\text {acc }}-\log M_{\star}$ plot. To generate this plot we use the results from the Siess et al. (2000) tracks for masses $\geq 0.1 M_{\odot}$ and those of Baraffe et al. (2015) for the three objects with lower values (see discussion in Sect. 4.1 of Alcalá et al. 2017, for the similarities of the tracks depending on mass).

The plot shows that the accretion properties of MY Lup, after the new $L_{\text {acc }}$ and $\dot{M}_{\text {acc }}$ estimate based on HST data (values in bold characters in Table A.1), are similar to those of transitional and Class II discs of similar mass. The general conclusion on the bi-modal behaviour of the $\log \dot{M}_{\mathrm{acc}}-\log M_{\star}$ relationship remains basically the same as in Alcalá et al. (2017) and Manara et al.

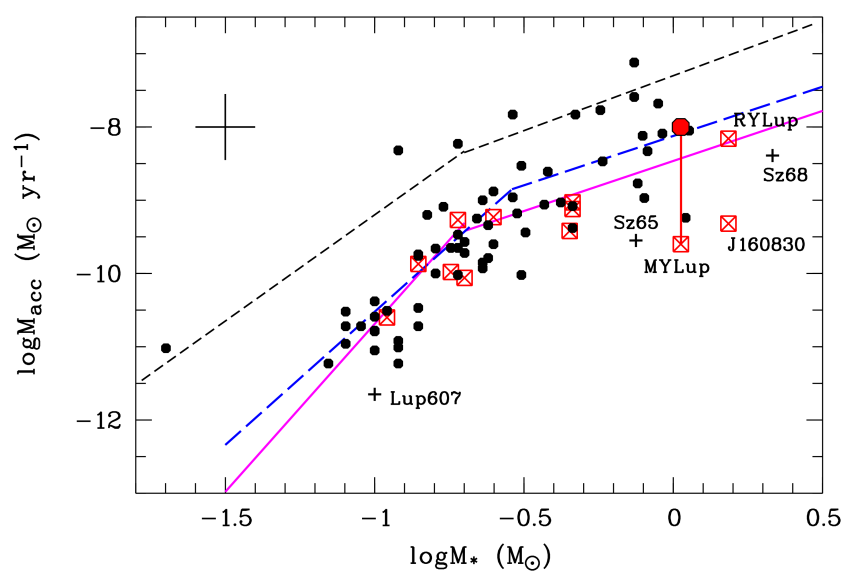

Fig. A.1. Mass accretion rate as a function of stellar mass for the Lupus sample of Alcalá et al. (2017) after revisiting the stellar and accretion parameters adopting the Gaia DR2 distances. The transitional discs are shown with red crossed squares. The average errors in $\log M_{\star}$ and $\log \dot{M}_{\text {acc }}$ are shown in the upper left. The symbols corresponding to MY Lup, SST c2dJ160830.7-382 827 and RY Lup, the latter also investigated with HST (Arulanantham et al. 2018), are labelled. The big red dot represents the corrected value of $\dot{M}_{\text {acc }}$ for the former, with the vertical red line indicating the corresponding vertical shift. The other three objects classified as weak or negligible accretors (see Sect. 1) are plotted with plus symbols and are also labelled. Sub-luminous objects are not plotted. The black dashed line shows the double power law theoretically predicted by Vorobyov \& Basu (2009), and the continuous magenta lines represent the fits to the data as in Eqs. (4) and (5) of Alcalá et al. (2017); the long-dashed blue line shows the robust double-linear fit following the prescription by Manara et al. (2017a).

(2017a). Interestingly, the transitional discs fall in general in the lower envelope of the distribution of points in the diagram. 
Table A.1. Revisited stellar and accretion properties of Lupus YSOs.

\begin{tabular}{|c|c|c|c|c|c|c|c|c|c|c|c|}
\hline Object & SpT & $\begin{array}{l}T_{\text {eff }} \\
(\mathrm{K})\end{array}$ & $\begin{array}{c}A_{\mathrm{V}} \\
(\mathrm{mag})\end{array}$ & $\begin{array}{c}d \\
(\mathrm{pc})\end{array}$ & $\begin{array}{l}L_{\star} \\
\left(L_{\odot}\right)\end{array}$ & $\begin{array}{c}\log L_{\mathrm{acc}} \\
\left(L_{\odot}\right)\end{array}$ & $\begin{array}{c}M_{\star}(\mathrm{B} 15) \\
\left(M_{\odot}\right)\end{array}$ & $\begin{array}{c}\log \dot{M}_{\mathrm{acc}}(\mathrm{B} 15) \\
\left(M_{\odot} \mathrm{yr}^{-1}\right)\end{array}$ & $\begin{array}{c}M_{\star}(\mathrm{S} 00) \\
\left(M_{\odot}\right)\end{array}$ & $\begin{array}{c}\log \dot{M}_{\mathrm{acc}}(\mathrm{S} 00) \\
\left(M_{\odot} \mathrm{yr}^{-1}\right)\end{array}$ & Notes \\
\hline Sz66 & M3 & 3415 & 1.00 & 157 & 0.22 & -1.76 & 0.29 & -8.50 & 0.31 & -8.53 & \\
\hline АKC2006-19 & M5 & 3125 & 0.00 & 153 & 0.02 & -4.08 & 0.14 & -10.97 & 0.12 & -10.92 & \\
\hline Sz69 & M4.5 & 3197 & 0.00 & 155 & 0.09 & -2.77 & 0.20 & -9.48 & 0.19 & -9.47 & \\
\hline Sz71 & M1.5 & 3632 & 0.50 & 156 & 0.33 & -2.17 & 0.41 & -9.02 & 0.42 & -9.03 & \\
\hline Sz72 & M2 & 3560 & 0.75 & 156 & 0.27 & -1.77 & 0.37 & -8.60 & 0.38 & -8.61 & \\
\hline Sz73 & $\mathrm{K} 7$ & 4060 & 3.50 & 157 & 0.46 & -0.96 & 0.78 & -8.12 & 0.79 & -8.12 & \\
\hline Sz74 & M3.5 & 3342 & 1.50 & 159 & 1.16 & -1.45 & 0.30 & -7.81 & 0.29 & -7.83 & \\
\hline Sz83 & $\mathrm{K} 7$ & 4060 & 0.00 & 160 & 1.49 & -0.25 & 0.67 & -7.08 & 0.74 & -7.12 & \\
\hline Sz84 & M5 & 3125 & 0.00 & 153 & 0.13 & -2.68 & 0.17 & -9.23 & 0.19 & -9.27 & $\mathrm{td}$ \\
\hline Sz130 & M2 & 3560 & 0.00 & 160 & 0.18 & -2.14 & 0.39 & -9.09 & 0.37 & -9.06 & \\
\hline Sz88A & M0 & 3850 & 0.25 & 158 & 0.31 & -1.40 & 0.61 & -8.49 & 0.58 & -8.47 & \\
\hline Sz88B & M4.5 & 3197 & 0.00 & 159 & 0.07 & -3.30 & 0.20 & -10.05 & 0.19 & -10.02 & \\
\hline Sz91 & M1 & 3705 & 1.20 & 159 & 0.20 & -2.00 & 0.51 & -9.07 & 0.46 & -9.03 & $\mathrm{td}$ \\
\hline Lup713 & M5.5 & 3057 & 0.00 & 174 & 0.02 & -3.62 & 0.11 & -10.40 & 0.10 & -10.38 & \\
\hline Lup604s & M5.5 & 3057 & 0.00 & 160 & 0.04 & -3.89 & 0.12 & -10.56 & 0.11 & -10.51 & \\
\hline Sz97 & M4 & 3270 & 0.00 & 158 & 0.11 & -3.11 & 0.24 & -9.88 & 0.23 & -9.85 & \\
\hline Sz99 & M4 & 3270 & 0.00 & 159 & 0.05 & -2.80 & 0.23 & -9.73 & 0.19 & -9.65 & \\
\hline Sz100 & M5.5 & 3057 & 0.00 & 137 & 0.08 & -3.33 & 0.14 & -9.87 & 0.14 & -9.87 & $\mathrm{td}$ \\
\hline Sz103 & M4 & 3270 & 0.70 & 160 & 0.12 & -2.60 & 0.23 & -9.33 & 0.24 & -9.34 & \\
\hline Sz104 & M5 & 3125 & 0.00 & 166 & 0.07 & -3.36 & 0.16 & -10.03 & 0.16 & -10.00 & \\
\hline Lup706 & M7.5 & 2795 & 0.00 & 159 & 0.002 & -5.00 & 0.06 & -11.90 & & & sl, ost \\
\hline Sz106 & M0.5 & 3777 & 1.00 & 162 & 0.06 & -2.68 & 0.57 & -10.07 & 0.49 & -10.00 & sl \\
\hline Par-Lup3-3 & M4 & 3270 & 2.20 & 159 & 0.15 & -3.10 & 0.23 & -9.77 & 0.24 & -9.79 & \\
\hline Par-Lup3-4 & M4.5 & 3197 & 0.00 & 151 & 0.002 & -4.35 & 0.17 & -11.81 & 0.16 & -11.78 & sl \\
\hline Sz110 & M4 & 3270 & 0.00 & 160 & 0.18 & -2.20 & 0.23 & -8.84 & 0.25 & -8.88 & \\
\hline Sz111 & M1 & 3705 & 0.00 & 158 & 0.21 & -2.40 & 0.51 & -9.47 & 0.45 & -9.42 & $\mathrm{td}$ \\
\hline Sz112 & M5 & 3125 & 0.00 & 160 & 0.12 & -3.39 & 0.17 & -9.94 & 0.18 & -9.98 & td \\
\hline Sz113 & M4.5 & 3197 & 1.00 & 163 & 0.04 & -2.28 & 0.19 & -9.12 & 0.17 & -9.09 & \\
\hline 2MASSJ16085953-3856275 & M8.5 & 2600 & 0.00 & 150 & 0.01 & -4.85 & 0.02 & -11.02 & & & ost \\
\hline SSTc2d160901.4-392512 & M4 & 3270 & 0.50 & 164 & 0.10 & -3.17 & 0.23 & -9.95 & 0.23 & -9.93 & \\
\hline Sz114 & M4.8 & 3175 & 0.30 & 162 & 0.21 & -2.68 & 0.19 & -9.17 & 0.22 & -9.25 & \\
\hline Sz115 & M4.5 & 3197 & 0.50 & 158 & 0.11 & -2.91 & 0.20 & -9.57 & 0.20 & -9.57 & \\
\hline Lup818s & M6 & 2990 & 0.00 & 157 & 0.02 & -4.31 & 0.08 & -10.96 & 0.10 & -11.05 & \\
\hline Sz123A & M1 & 3705 & 1.25 & 159 & 0.13 & -2.00 & 0.55 & -9.21 & 0.46 & -9.12 & $\mathrm{td}$ \\
\hline Sz123B & M2 & 3560 & 0.00 & 159 & 0.03 & -2.90 & 0.40 & -10.24 & 0.32 & -10.13 & sl \\
\hline SST-Lup3-1 & M5 & 3125 & 0.00 & 165 & 0.04 & -3.77 & 0.16 & -10.53 & 0.14 & -10.47 & \\
\hline Sz65 & K7 & 4060 & 0.60 & 155 & 0.89 & -2.57 & 0.70 & -9.52 & 0.75 & -9.55 & \\
\hline AKC2006-18 & M6.5 & 2935 & 0.00 & 149 & 0.01 & -4.60 & 0.07 & -11.23 & & & ost \\
\hline SSTc2dJ154508.9-341734 & M5.5 & 3060 & 5.50 & 155 & 0.06 & -1.77 & 0.14 & -8.36 & 0.12 & -8.32 & \\
\hline Sz68 & $\mathrm{K} 2$ & 4900 & 1.00 & 154 & 5.42 & -1.18 & & & 2.15 & -8.39 & obt \\
\hline SSTc2dJ154518.5-342125 & M6.5 & 2935 & 0.00 & 152 & 0.04 & -4.29 & 0.08 & -10.72 & 0.10 & -10.79 & \\
\hline Sz81A & M4.5 & 3200 & 0.00 & 160 & 0.25 & -2.44 & 0.19 & -8.92 & 0.23 & -9.00 & \\
\hline Sz81B & M5.5 & 3060 & 0.00 & 160 & 0.12 & -3.14 & 0.15 & -9.61 & 0.16 & -9.66 & \\
\hline Sz129 & $\mathrm{K} 7$ & 4060 & 0.90 & 162 & 0.43 & -1.13 & 0.78 & -8.30 & 0.82 & -8.33 & \\
\hline SSTc2dJ155925.2-423507 & M5 & 3125 & 0.00 & 147 & 0.02 & -4.42 & 0.14 & -11.29 & 0.12 & -11.23 & \\
\hline RYLup & $\mathrm{K} 2$ & 4900 & 0.40 & 159 & 1.87 & -0.85 & & & 1.53 & -8.16 & $\mathrm{td}, \mathrm{obt}$ \\
\hline SSTc2dJ160000.6-422158 & M4.5 & 3200 & 0.00 & 161 & 0.10 & -3.04 & 0.20 & -9.73 & 0.20 & -9.72 & \\
\hline SSTc2dJ160002.4-422216 & M4 & 3270 & 1.40 & 164 & 0.18 & -2.92 & 0.23 & -9.56 & 0.25 & -9.60 & \\
\hline SSTc2dJ160026.1-415356 & M5.5 & 3060 & 0.90 & 164 & 0.08 & -3.22 & 0.14 & -9.76 & 0.14 & -9.76 & \\
\hline MYLup & K0 & 5100 & 1.30 & 157 & 0.85 & -0.65 & 1.09 & -8.01 & 1.06 & -7.99 & $\mathrm{td} ?$ \\
\hline Sz131 & M3 & 3415 & 1.30 & 160 & 0.15 & -2.34 & 0.30 & -9.18 & 0.30 & -9.18 & \\
\hline Sz133 & $\mathrm{K} 5$ & 4350 & 1.80 & 153 & 0.07 & -1.78 & & & & & $\mathrm{sl}, \mathrm{bz}$ \\
\hline SSTc2dJ160703.9-391112 & M4.5 & 3200 & 0.60 & 159 & 0.003 & -5.40 & & & 0.16 & -12.76 & sl, obt \\
\hline Sz90 & K7 & 4060 & 1.80 & 160 & 0.42 & -1.79 & 0.78 & -8.96 & 0.80 & -8.97 & \\
\hline Sz95 & M3 & 3415 & 0.80 & 158 & 0.26 & -2.70 & 0.29 & -9.40 & 0.32 & -9.44 & \\
\hline Sz96 & M1 & 3705 & 0.80 & 157 & 0.42 & -2.51 & 0.45 & -9.37 & 0.46 & -9.38 & \\
\hline
\end{tabular}

Notes. td: transitional disc, sl: sub-luminous YSO, bz: sub-luminous object falling below the zero-age main sequence on the HR diagram, ost: outside mass range of the S00 tracks, obt: outside mass range of the B15 tracks. 
Table A.1. continued.

\begin{tabular}{|c|c|c|c|c|c|c|c|c|c|c|c|}
\hline Object & SpT & $\begin{array}{l}T_{\text {eff }} \\
(\mathrm{K})\end{array}$ & $\begin{array}{c}A_{\mathrm{V}} \\
(\mathrm{mag})\end{array}$ & $\begin{array}{c}d \\
(\mathrm{pc})\end{array}$ & $\begin{array}{l}L_{\star} \\
\left(L_{\odot}\right) \\
\end{array}$ & $\begin{array}{c}\log L_{\mathrm{acc}} \\
\left(L_{\odot}\right) \\
\end{array}$ & $\begin{array}{c}M_{\star}(\mathrm{B} 15) \\
\left(M_{\odot}\right)\end{array}$ & $\begin{array}{c}\log \dot{M}_{\mathrm{acc}}(\mathrm{B} 15) \\
\left(M_{\odot} \mathrm{yr}^{-1}\right)\end{array}$ & $\begin{array}{c}M_{\star}(\mathrm{S} 00) \\
\left(M_{\odot}\right) \\
\end{array}$ & $\begin{array}{c}\log \dot{M}_{\mathrm{acc}}(\mathrm{S} 00) \\
\left(M_{\odot} \mathrm{yr}^{-1}\right)\end{array}$ & Notes \\
\hline 2MASSJ16081497-3857145 & M5.5 & 3060 & 1.50 & 159 & 0.01 & -3.60 & 0.10 & -10.60 & 0.11 & -10.60 & td \\
\hline Sz98 & $\mathrm{K} 7$ & 4060 & 1.00 & 156 & 1.53 & -0.71 & 0.67 & -7.54 & 0.74 & -7.59 & \\
\hline Lup607 & M6.5 & 2935 & 0.00 & 175 & 0.05 & -5.02 & 0.09 & -11.60 & 0.10 & -11.65 & \\
\hline Sz102 & $\mathrm{K} 2$ & 4900 & 0.70 & 159 & 0.01 & -2.20 & & & & & $\mathrm{sl}, \mathrm{bz}$ \\
\hline SSTc2dJ160830.7-382827 & $\mathrm{K} 2$ & 4900 & 0.20 & 156 & 1.84 & -2.02 & & & 1.53 & -9.32 & $\mathrm{td}$ \\
\hline SSTc2dJ160836.2-392302 & K6 & 4205 & 1.70 & 154 & 1.15 & -1.03 & 0.83 & -8.04 & 0.92 & -8.09 & \\
\hline Sz108B & M5 & 3125 & 1.60 & 169 & 0.11 & -3.05 & 0.17 & -9.62 & 0.18 & -9.65 & \\
\hline 2MASSJ16085324-3914401 & M3 & 3415 & 1.90 & 168 & 0.21 & -3.25 & 0.29 & -10.00 & 0.31 & -10.02 & \\
\hline 2MASSJ16085373-3914367 & M5.5 & 3060 & 4.00 & 159 & 0.00 & -3.90 & 0.10 & -10.94 & 0.12 & -11.01 & \\
\hline 2MASSJ16085529-3848481 & M6.5 & 2935 & 0.00 & 158 & 0.05 & -4.31 & 0.09 & -10.72 & 0.10 & -10.78 & \\
\hline SSTc2dJ160927.0-383628 & M4.5 & 3200 & 2.20 & 159 & 0.07 & -1.50 & 0.20 & -8.25 & 0.19 & -8.23 & \\
\hline Sz117 & M3.5 & 3340 & 0.50 & 159 & 0.28 & -2.30 & 0.25 & -8.91 & 0.29 & -8.96 & \\
\hline Sz118 & $\mathrm{K} 5$ & 4350 & 1.90 & 164 & 0.72 & -1.97 & 1.04 & -9.21 & 1.10 & -9.24 & \\
\hline 2MASSJ16100133-3906449 & M6.5 & 2935 & 1.70 & 193 & 0.19 & -3.43 & & & 0.14 & -9.74 & obt \\
\hline SSTc2dJ161018.6-383613 & M5 & 3125 & 0.50 & 159 & 0.04 & -4.00 & 0.15 & -10.76 & 0.14 & -10.72 & \\
\hline SSTc2dJ161019.8-383607 & M6.5 & 2935 & 0.00 & 159 & 0.04 & -4.10 & 0.08 & -10.52 & 0.10 & -10.59 & \\
\hline SSTc2dJ161029.6-392215 & M4.5 & 3200 & 0.90 & 163 & 0.11 & -3.38 & 0.20 & -10.05 & 0.20 & -10.06 & td \\
\hline SSTc2dJ161243.8-381503 & M1 & 3705 & 0.80 & 160 & 0.39 & -2.19 & 0.45 & -9.07 & 0.46 & -9.08 & \\
\hline SSTc2dJ161344.1-373646 & M5 & 3125 & 0.60 & 160 & 0.04 & -2.49 & 0.16 & -9.24 & 0.15 & -9.20 & \\
\hline Sz75 & K6 & 4205 & 0.70 & 152 & 1.48 & -0.69 & 0.80 & -7.63 & 0.89 & -7.68 & \\
\hline Sz76 & M4 & 3270 & 0.20 & 160 & 0.18 & -2.55 & 0.23 & -9.18 & 0.25 & -9.23 & td \\
\hline Sz77 & $\mathrm{K} 7$ & 4060 & 0.00 & 155 & 0.59 & -1.67 & 0.75 & -8.76 & 0.76 & -8.77 & \\
\hline RXJ1556.1-3655 & M1 & 3705 & 1.00 & 158 & 0.26 & -0.85 & 0.49 & -7.85 & 0.47 & -7.83 & \\
\hline Sz82 & K5 & 4350 & 0.90 & 159 & 2.60 & -1.05 & 0.95 & -7.98 & 1.13 & -8.05 & \\
\hline EXLup & M0 & 3850 & 1.10 & 158 & 0.76 & -0.91 & 0.53 & -7.74 & 0.57 & -7.77 & \\
\hline
\end{tabular}

